\title{
Efficacy of Combined Therapy with Drug-Eluting Beads-Transcatheter Arterial Chemoembolization Followed by Conventional Transcatheter Arterial Chemoembolization for Unresectable Hepatocellular Carcinoma: A Multi-Center Study
}

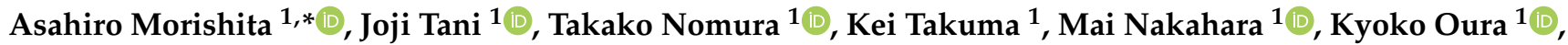 \\ Tomoko Tadokoro ${ }^{1}$, Koji Fujita ${ }^{1}{ }^{\mathbb{D}}$, Tingting Shi ${ }^{1}{ }^{\mathbb{D}}$, Hiroki Yamana ${ }^{1}$, Takanori Matsui ${ }^{1}$, Tadayuki Takata ${ }^{1}$, \\ Takayuki Sanomura ${ }^{2}$, Yoshihiro Nishiyama ${ }^{2}$, Takashi Himoto ${ }^{3}$, Tetsu Tomonari ${ }^{4}$, Akio Moriya ${ }^{5}$, \\ Tomonori Senoo ${ }^{6}$, Koichi Takaguchi ${ }^{6}$ and Tsutomu Masaki ${ }^{1}$
}

check for

updates

Citation: Morishita, A.; Tani, J.; Nomura, T.; Takuma, K.; Nakahara, M.; Oura, K.; Tadokoro, T.; Fujita, K.; Shi, T.; Yamana, H.; et al. Efficacy of Combined Therapy with Drug-Eluting Beads-Transcatheter Arterial Chemoembolization Followed by Conventional

Transcatheter Arterial

Chemoembolization for Unresectable Hepatocellular Carcinoma: A Multi-Center Study. Cancers 2021, 13, 4605. https://doi.org/10.3390/ cancers13184605

Academic Editor: Samuel C. Mok

Received: 12 July 2021

Accepted: 9 September 2021

Published: 14 September 2021

Publisher's Note: MDPI stays neutral with regard to jurisdictional claims in published maps and institutional affiliations.

Copyright: (c) 2021 by the authors. Licensee MDPI, Basel, Switzerland. This article is an open access article distributed under the terms and conditions of the Creative Commons Attribution (CC BY) license (https:/ / creativecommons.org/licenses/by/ $4.0 /$ )
1 Department of Gastroenterology and Neurology, Kagawa University, Kita-gun 761-0793, Japan; georget@med.kagawa-u.ac.jp (J.T.); takako-n@med.kagawa-u.ac.jp (T.N.); k-takuma@med.kagawa-u.ac.jp (K.T.); m-nakahara@med.kagawa-u.ac.jp (M.N.); kyoko_oura@med.kagawa-u.ac.jp (K.O.); t-nishioka@med.kagawa-u.ac.jp (T.T.); 92m7v9@med.kagawa-u.ac.jp (K.F.); shitingtingc@med.kagawa-u.ac.jp (T.S.); yamanahiroki@med.kagawa-u.ac.jp (H.Y.); s16d729@stu.kagawa-u.ac.jp (T.M.); takata.tadayuki@kagawa-u.ac.jp (T.T.); tmasaki@med.kagawa-u.ac.jp (T.M.)

2 Department of Radiology, Kagawa University, Kita-gun 761-0793, Japan; sanomura@med.kagawa-u.ac.jp (T.S.); nisiyosi@med.kagawa-u.ac.jp (Y.N.)

3 Department of Medical Technology, Kagawa Prefectural University of Health Sciences, Takamatsu 761-0123, Japan; himoto@chs.pref.kagawa.jp

4 Department of Gastroenterology and Oncology, Institute of Biomedical Sciences, Graduate School of Medicine, Tokushima University, Tokushima 770-8503, Japan; tetsu.tomonari@gmail.com

5 Department of Gastroenterology, Mitoyo General Hospital, Kanonji 769-1695, Japan; amoriya@mitoyo-hosp.jp

6 Department of Hepatology, Kagawa Prefectural Central Hospital, Takamatsu 760-8557, Japan; t-senoo@chp-kagawa.jp (T.S.); k.takaguchi@chp-kagawa.jp (K.T.)

* Correspondence: asahiro@med.kagawa-u.ac.jp; Tel.: +81-87-891-2156; Fax: +81-87-891-2158

Simple Summary: Drug-eluting beads-transcatheter chemoembolization (DEB-TACE) has recently been performed. However, local recurrence of HCC at the tumor margins is often observed. Conventional transcatheter chemoembolization (cTACE) comprises accumulating lipiodol-containing anticancer drugs into the drainage area, which is the first invasive site of hepatocellular carcinoma (HCC). We evaluate the therapeutic effect of DEB-TACE followed by cTACE in patients with intermediate stage HCC. HCC patients were divided into two groups: one group received DEB-TACE followed by cTACE (cTACE group) and the other group received only DEB-TACE (non-cTACE group). The complete response (CR) rate was significantly higher in the cTACE group than in the non-TACE group. The only factor that increased the complete response rate in the cTACE group was the number of tumors. The overall survival (OS) rate of CR patients was higher than that of non-CR patients in the cTACE group. CTACE group adverse events included severe thrombocytopenia but only in one patient. The combined therapy with DEB-TACE followed by cTACE may be useful for HCC patients.

Abstract: EB-TACE has recently been performed because of its lower hepatotoxicity compared to CTACE in less advanced HCC. However, local recurrence at the tumor margins is often observed after DEB-TACE. cTACE involves filling the intratumoral sinusoids with lipiodol-containing anticancer drugs and accumulating in the drainage area, which is the first site of HCC recurrence. The aim of this study is to evaluate the therapeutic effect of DEB-TACE followed by cTACE in HCC patients. Between 2014 and 2020, 65 patients with Barcelona clinic liver cancer (BCLC) stage B (intermediate stage) of HCC were enrolled and divided into two groups: one group received DEB-TACE followed by cTACE (cTACE group) and the other group received only DEB-TACE (non-cTACE group). Sixty-five patients were medically followed. The median observation time was $14 \pm 13.1$ months after the first 
DEB-TACE and outcomes were analyzed for multiple factors. Results: The complete response rate was significantly higher in the cTACE group than in the non-TACE group. The analysis showed that the only factor that increased the CR rate in the CTACE group was the total tumor number (less than four). The OS rate of CR patients was higher than that of non-CR patients in the CTACE group. Adverse events in the cTACE group included severe thrombocytopenia but only in one of twenty-seven patients. Conclusions: The combined therapy with DEB-TACE followed by cTACE may be a new effective therapeutic strategy for the intermediate stage of HCC patients.

Keywords: DEB-TACE; cTACE; Barcelona Clinic Liver Cancer staging system; combination therapy; HCC

\section{Introduction}

HCC is the most common cancer [1,2] and the fourth-leading cause of death worldwide [3-6]. Patient prognosis remains poor due to a lack of effective therapy [7]. While surgery is the most effective therapy for HCC [8], most patients are diagnosed at advanced stages precluding surgical therapy. For these patients, the conventional therapies are CTACE [9], radiofrequency ablation [10], molecular-targeting drugs, or these in combination therapies $[2,11]$.

The Barcelona Clinic Liver Cancer Staging System [12] is widely used to evaluate the staging and consequential treatment of HCC. To treat the intermediate stage of HCC, CTACE is often performed [2,13]. Recently, drug-eluting beads-transcatheter chemoembolization (DEB-TACE) has been recognized as an alternative therapy for patients with advanced, unresectable liver cancer [14,15]. During DEB-TACE, the patient is injected with chemotherapy-loaded microbeads which embolize the arteries that feed the tumor; the drug-loaded beads slowly release a chemotherapeutic agent into the tumor with a systemic drug concentration peak of less than that in CTACE [16]. DEB-TACE reduces the risk of drug-related adverse events such as post-embolization syndrome [1,17]. Zhiyi et al. demonstrated that DEB-TACE treatment achieved a 19.9\% complete response (CR) rate and a 79.6\% objective response rate [18]. However, after DEB-TACE, residual areas are often found at the tumor margins, at which increased local recurrence can cause serious damage to the patient. In other words, this residual area, at which drug-eluting beads cannot reach, reduces the CR rate. Therefore, CTACE [19], which is effective for this residual region, is performed as a second line treatment. The aim of our study was to evaluate the efficacy and safety of combination therapy with DEB-TACE followed by cTACE in a short term for the treatment of the intermediate stage of unresectable liver cancer.

\section{Methods}

\subsection{Patient Selection and Eligibility}

This multiple-institution retrospective study was approved by the Institutional Ethics Committee of the Kagawa University, Faculty of Medicine (Kita-gun, Japan), in accordance with the Declaration of Helsinki (approval number 2019-271, approved on March 31, 2020). Sixty-five patients with HCC who underwent DEB-TACE between 2014 and 2020 at the Kagawa University Hospital (Kita-gun, Japan), Tokushima University Hospital (Tokushima, Japan), Mitoyo General Hospital (Mitoyo-shi, Japan), and Kagawa Prefectural Central Hospital (Takamatsu, Japan) were examined for this retrospective study. The requirement for informed consent from the participants was waived because of the retrospective nature of the study. The study inclusion criteria were: (i) patients diagnosed with the intermediate stage of hepatocellular carcinoma in accordance with the Barcelona Clinic Liver Cancer Staging System [2]; (ii) patients over 18 years old; and (iii) patients who had received DEB-TACE treatment. The exclusion criteria were: (i) patients with advanced stage liver cancer; (ii) patients who were lost to follow-up; (iii) patients with liver or renal failure; and (iv) patients with an allergy to chemoembolization reagents. The patient data collected in 
our study also included sex, age, etiology of cirrhosis, the Child-Pugh score, clinical tumor stage (cStage), macroscopic classification, up-to-7 criteria, alpha fetoprotein (AFP), the third electrophoretic form of lentil lectin-reactive AFP (AFP-L3) [20], and des-gamma-carboxy prothrombin (DCP) [21] (Table 1).

Table 1. Baseline characteristics of the patients examined in the study.

\begin{tabular}{|c|c|c|c|}
\hline Parameters & $n=27, \operatorname{cTACE}(+)$ & $n=38$, cTACE $(-)$ & $p$-Values \\
\hline Age, median (range) & $75(54-89)$ & $78(54-90)$ & 0.3286 \\
\hline Sex (male/female) & $18 / 9$ & $31 / 7$ & 0.1375 \\
\hline Etiology (HBV or HCV/NBNC) & $11 / 16$ & $23 / 15$ & 0.1155 \\
\hline Child-Pugh score $(5,6 / 7$, or 8$)$ & $21 / 6$ & $23 / 15$ & 0.1428 \\
\hline cStage (II/III) & $8 / 19$ & $19 / 19$ & 0.1288 \\
\hline Tumor number $(<4 / 4 \leq)$ & $20 / 7$ & $34 / 4$ & 0.1027 \\
\hline $\begin{array}{l}\text { Maximum tumor size }(\mathrm{mm}), \\
\text { median (range) }\end{array}$ & $59(30-131)$ & $69(12-200)$ & 0.7136 \\
\hline $\mathrm{MC}(\mathrm{SN}$ or SNE/CMN) & $23 / 4$ & $31 / 7$ & 0.7024 \\
\hline up-to-7 criteria (IN/OUT) & $14 / 13$ & $26 / 12$ & 0.1760 \\
\hline AFP (ng/mL), median (range) & $9192(4-149,280)$ & $19,672(2-250,434)$ & 0.9038 \\
\hline AFP-L3 (\%), median (range) & $19(0.1-82)$ & $32(0.5-86)$ & 0.6389 \\
\hline DCP (mAU/mL), median (range) & $9594(30-68,034)$ & $39,178(8-816,823)$ & 0.3717 \\
\hline
\end{tabular}

Abbreviations: $\mathrm{HBV}$, hepatitis $\mathrm{B}$ virus; $\mathrm{HCV}$, hepatitis $\mathrm{C}$ virus; cStage, clinical tumor stage; $\mathrm{MC}$, macroscopic classification; SN, single nodular type; SNE, single nodular type with extranodular growth; CMN, confluent multinodular type; AFP, $\alpha$-fetoprotein; and DCP, des- $\gamma$ carboxy prothrombin.

\subsection{DEB-TACE Procedures}

After the supernatant was extracted from one bottle of drug-eluting beads (DC beads; Eisai Co. Ltd., Tokyo, Japan), one vial ( $2 \mathrm{~mL}$ ) of drug-eluting beads with diameters of 100-300 $\mu \mathrm{m}$ was loaded with 50 mg of epirubicin (Nippon Kayaku Co. Ltd., Tokyo, Japan) and diluted 10 times with iopamidol $300 \mathrm{mgI} / \mathrm{mL}$ (Iopamiron 300; Bayer Schering Pharma, Osaka, Japan) [22]. Each drug-eluting bead was transferred to a 20-mL syringe in the significant diffusion state and injected at $1 \mathrm{~mL} / \mathrm{min}$. A microcatheter that was $130 \mathrm{~cm}$ in length (Progreat ${ }^{\circledR}$; Terumo Co. Ltd., Tokyo, Japan) was used. The outer diameters of the catheter tip and shaft were 1.7 and $2.8 \mathrm{Fr}$, and the inner diameters were 0.016 and 0.026 inches. This procedure was conducted at room temperature. Angiography was performed to detect the tumor-supplying vessels and both the microcatheter and microwire were super-selectively catheterized into the tumor-supplying vessels for embolization. The embolization was discontinued after the flow of the contrast agent stopped. Within 5 min of the chemotherapeutic agent delivery, another angiography was performed to determine if the [blushed/tinted] tumor was still visible and if so, the embolization procedure was repeated. One or two vials (maximum two vials) of DC beads were used in all procedures. Gelpart (1 mg; Nippon Kayaku Co. Ltd., Tokyo, Japan) was administered during arterial embolization if a vascular lake was detected in the tumor.

\section{3. cTACE Procedures and Timing of Additional cTACE}

Angiography was performed to detect the tumor-supplying vessels and percutaneous femoral arterial puncture was performed using the Seldinger technique under topical anesthesia [23]. Subsequently, the microcatheter (1.7Fr; Breakthrough ${ }^{\mathrm{TM}}$, Boston Scientific, Marlborough, MA, USA) and microwire (0.016 inch; ASAHI Meister, ASAHI INTECC Co., ltd., Seto, Japan) were super-selectively catheterized into the tumor-supplying vessels for the delivery of the chemotherapeutic reagent, namely a solution of cisplatin (Nichi-iko Pharma Co. Ltd., Toyama, Japan), miriplatin (Dainippon Sumitomo Pharma Co., Ltd., Osaka, Japan) or epirubicin and lipiodol (Guerbet Japan Co. Ltd., Tokyo, Japan). Under radiographic guidance, the infusion was discontinued when the flow of lipiodol stopped. Another angiography was performed to ensure that lipiodol had been deposited and to confirm adequate infusion. Gelpart (1 mg; Nippon Kayaku Co. Ltd., Tokyo, Japan) 
was administered after the transarterial infusion of lipiodol with the chemotherapeutic reagents until adequate embolization. Second or third CTACE was performed in 2 months after DEB-TACE.

\subsection{Treatment Outcome Assessment Criteria}

Treatment outcomes were assessed within 1-3 months after the first cycle of DEBTACE and again after the second or third cycle of cTACE according to imaging results and the Response Evaluation Criteria in Cancer of the Liver version 5.0, as follows. (i) A complete response: the loss of any intratumoral arterial enhancement in all target nodules; (ii) a partial response: at least a 30\% decrease in the sum of the diameters of viable (enhancement in the arterial phase) target nodules relative to the baseline sum of the diameters of target nodules; (iii) a stable disease: absence of partial response or progressive disease; (iv) a progressive disease: an increase of at least $20 \%$ in the sum of the diameters of viable (enhancing) target nodules relative to the smallest sum of the diameters of viable (enhancing) target nodules recorded since treatment started; (v) an objective response rate: the percentage of patients who achieved a complete response or partial response; and (vi) a disease control rate: the percentage of patients who achieved a complete response, partial response, or stable disease [24].

\subsection{Liver Function and Safety Assessment}

Liver function was assessed using liver function-related laboratory parameters including albumin, total bilirubin, alanine aminotransferase, and aspartate aminotransferase. All adverse events including pain, fever, nausea, and vomiting were recorded using the Common Terminology Criteria for Adverse Events (CTCAE c5.0) [25].

\subsection{Statistical Analyses}

GraphPad Prism version 8.4.2 (GraphPad Software, San Diego, CA, USA) was used for the statistical analyses. Data are presented as count (\%), mean \pm standard deviation, or median (25-75th). A comparison between the two treatment groups was performed by the chi-square test. The Student's $t$-test was used to compare numerical data for each group. A value of $p<0.05$ was considered significant. Univariate analyses for continuous variables were undertaken using the Student's $t$-test, paired $t$-test, and one-way ANOVA. For the analysis of categorical variables, the Mann-Whitney U test, Fisher's exact test, chisquared test, proportional hazard model test, and Gray's test with log-rank test results were performed. A multivariate analysis was performed using the Cox proportional hazards model and was applied only to variables that were statistically $p<0.05$ in the univariate analysis. A survival analysis was performed using the Kaplan-Meier method.

\section{Results}

\subsection{Course of Treatment}

As shown in Figure 1, after seventy-one study participants received their first DEBTACE treatment, six patients were excluded from the study due to having the BCLC C stage of liver cancer or loss to follow-up. The final study population consisted of 65 patients. Of these, nine patients received a second DEB-TACE treatment. Three patients from this group of nine received a third cTACE treatment. Twenty-seven patients received a second or third cTACE treatment: fourteen patients using cisplatin, eleven patients using miriplatin, and two patients using epirubicin. Thirty-eight patients underwent non-cTACE treatment, including six patients with a second DEB-TACE treatment.

\subsection{Patient Characteristics}

The baseline characteristics of the patients with HCC are summarized in Table 1. There were no significant differences between the cTACE and non-cTACE groups with respect to gender, age, etiology (HBV or $\mathrm{HCV} / \mathrm{NBNC}$ ), Child-Pugh score (5 or 6/7 or 8 ), clinical stage (II or III/IVA or IVB), tumor number $(<4 / 4 \leq)$, maximum tumor size, 
MC (SN or SNE/CMN), up-to-7 criteria (IN/OUT), AFP, AFP-L3, or DCP. The cTACE group consisted of eighteen male and nine female patients with a median age of seventyfive (range: 54-89) years. The non-cTACE group consisted of thirty-one male and seven female patients with a median age of seventy-eight (range: 54-90) years (Table 1). The number of patients with tumors $<4 \mathrm{~cm}$ and $\geq 4 \mathrm{~cm}$ in size were twenty $(74.1 \%)$ and seven $(25.9 \%)$ in the cTACE group, respectively, and thirty-four $(89.5 \%)$ and four $(10.5 \%)$ in the non-cTACE group, respectively (Table 1). The median tumor size was $59 \mathrm{~mm}$ (range: $30-131)$ in the cTACE group and $69 \mathrm{~mm}$ (range: 12-200) in the non-cTACE group. In the CTACE group, twenty-three patients $(85.2 \%)$ had single nodular-type lesions or those with extranodular growth and four patients $(14.8 \%)$ had confluent multinodular-type lesions. In the non-cTACE group, thirty-one patients $(81.6 \%)$ had single nodular-type lesions with or without extranodular growth and seven patients $(18.4 \%)$ had confluent multinodular-type lesions (Table 1).

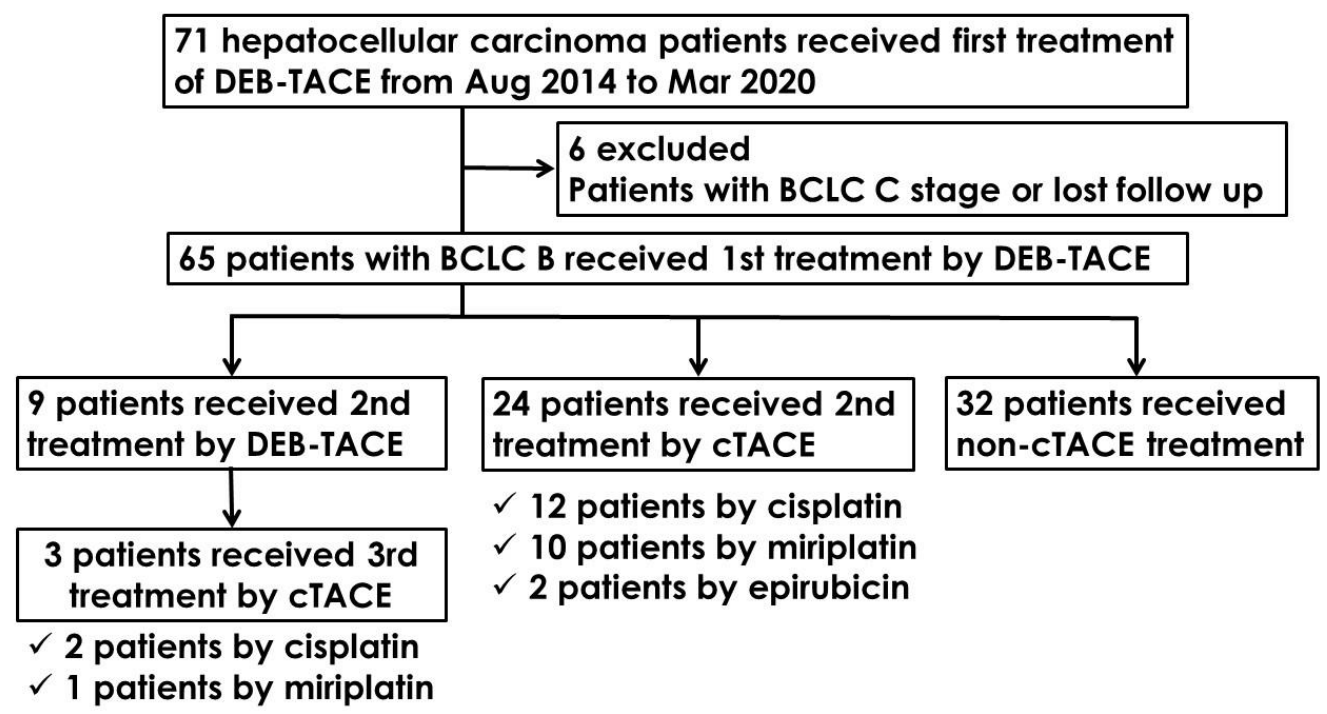

Figure 1. Flow chart of treatment received by the 65 patients enrolled in our study.

\subsection{Treatment Responses}

In the cTACE group, after the first cycle of DEB-TACE, no patient and 26 patients (96.3\%) achieved a complete response and partial response (Figure 2a-c), respectively, resulting in an overall response rate of $96.3 \%$ and a disease control rate of $100 \%$ (Table 2). In the non-cTACE group, the numbers of patients who achieved a complete response and partial response were $1(2.6 \%)$ and $32(84.2 \%)$, respectively, resulting in an overall response rate of $86.8 \%$ and a disease control rate of $86.8 \%$ (Table 2). No statistically significant difference was detected between the CTACE and non-cTACE groups after the first DEB-TACE.

We also examined the effect of additional cTACE on the treatment outcome of the first DEB-TACE or second DEB-TACE. In the non-cTACE group, four patients $(10.5 \%)$ achieved a complete response and twenty-four patients $(63.2 \%)$ achieved a partial response after the first DEB-TACE or second DEB-TACE, while fourteen patients (51.9\%) and eight patients $(29.6 \%)$ achieved a complete response (Figure $2 \mathrm{~d}, \mathrm{e}, \mathrm{f}$ ) and partial response, respectively, in the cTACE group (Table 3). Interestingly, the complete response rate in the cTACE group was significantly higher than that in the non-cTACE group $(p=0.0002)$. The overall response rate and disease control rate were not significantly different between the groups (both $p>0.05$; Table 3 ). 


\section{First DEB-TACE}

\section{Before TACE}

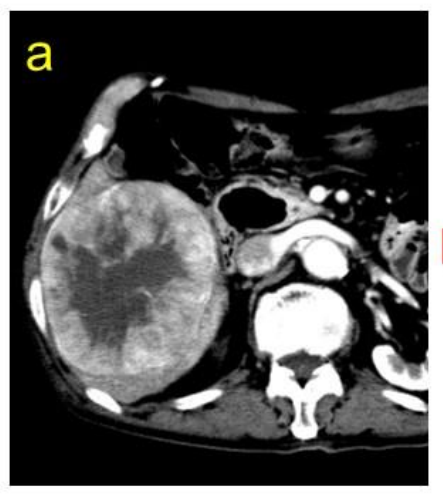

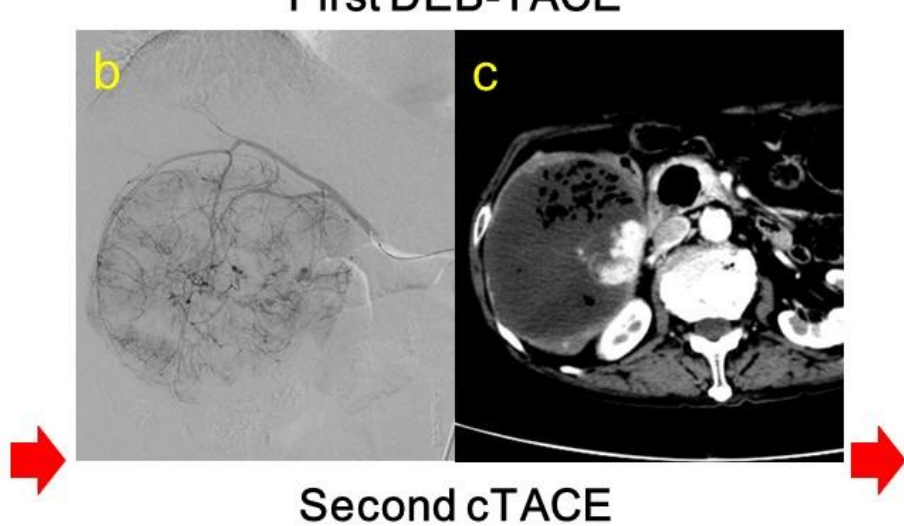

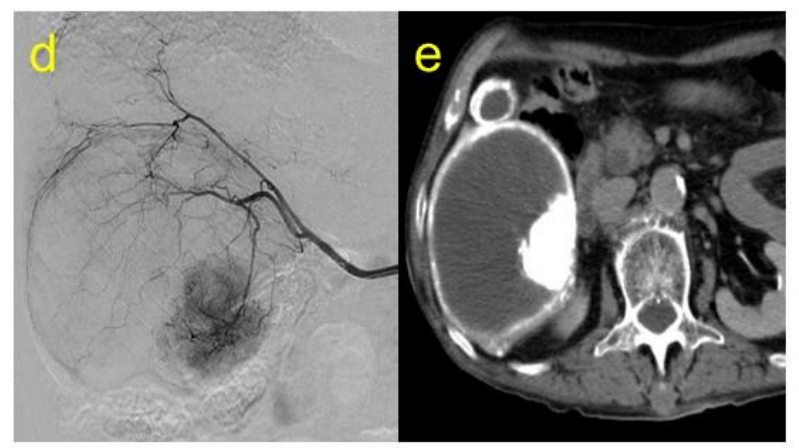

After TACE

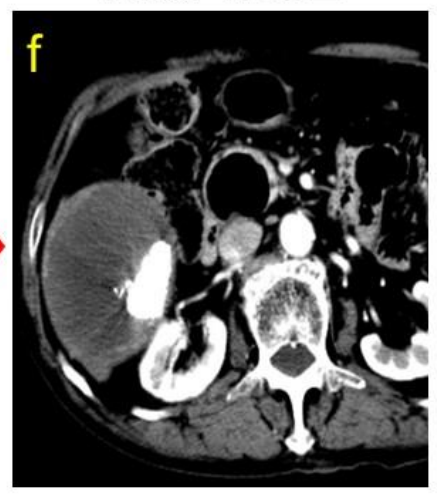

Figure 2. Radiographic images showing progress of treatment in 54-year-old female study participant with hepatocellular carcinoma: (a) dynamic computer tomography image from early treatment phase before transcatheter arterial chemoembolization (TACE) procedures; (b) angiographic image taken during the first drug-eluting beads-transcatheter chemoembolization (DEB-TACE) procedure; (c) dynamic computer tomography image from the early treatment phase a month after the first DEB-TACE procedure; (d) angiographic image taken during the second conventional TACE (cTACE) procedure; (e) regular computer tomography image taken immediately after the second cTACE procedure; and (f) dynamic computer tomography image taken a month after the second CTACE procedure.

Table 2. Treatment response to first DEB-TACE.

\begin{tabular}{cccc}
\hline Parameters & cTACE $(+), \boldsymbol{n}=\mathbf{2 7}$ & cTACE $(-), \boldsymbol{n}=\mathbf{3 8}$ & $\boldsymbol{p}$-Values \\
\hline Complete response (CR) & $0(0)$ & $1(2.6)$ & 0.3959 \\
Partial response (PR) & $26(96.3)$ & $32(84.2)$ & 0.1214 \\
Stable disease (SD) & $1(0)$ & $0(0)$ & 0.2319 \\
Progressive disease (PD) & $0(0)$ & $5(13.2)$ & 0.0500 \\
Overall response rate (ORR) & $26(96.3)$ & $33(86.8)$ & 0.1944 \\
Disease control rate (DCR) & $27(100)$ & $33(86.8)$ & 0.0500 \\
\hline
\end{tabular}

Data are presented as count $n(\%)$.

Table 3. Treatment response to additional cTACE.

\begin{tabular}{cccc}
\hline Parameters & cTACE $(+), \boldsymbol{n = 2 7}$ & cTACE $(-), \boldsymbol{n}=\mathbf{3 8}$ & $\boldsymbol{p}$-Values \\
\hline Complete response (CR) & $14(51.9)$ & $4(10.5)$ & ${ }^{*} 0.0002$ \\
Partial response (PR) & $8(29.6)$ & $24(63.2)$ & ${ }^{*} 0.0077$ \\
Stable disease (SD) & $1(3.7)$ & $2(5.3)$ & 0.7678 \\
Progressive disease (PD) & $4(14.8)$ & $8(21.1)$ & 0.5230 \\
Overall response rate (ORR) & $22(81.5)$ & $28(73.7)$ & 0.4622 \\
Disease control rate (DCR) & $23(85.2)$ & $30(78.9)$ & 0.5230 \\
\hline
\end{tabular}

Data are presented as count $n(\%)$. cTACE $(-)$ excluded second DEB-TACE $(* p<0.05)$. 


\subsection{Factors Contributing to Complete Response to DEB-TACE Followed by cTACE}

Our analysis of various factors related to the complete response to DEB-TACE followed by cTACE is shown in Table 4. Based on our analysis, the following factors did not contribute: age, Child-Pugh score, etiology, tumor size, MC, AFP, AFP-L3, and DCP. No difference between cTACE with cisplatin and that with miriplatin/epirubicin contributed to the complete response. Remarkably, tumor number alone $(<4 / \geq 4)$ contributed to the $\mathrm{CR}$ rate, in contrast to non-CR [partial response (PR), stable disease (SD), and progressive disease (PD)] rate (Table 4). These results suggest that DEB-TACE followed by cTACE is highly effective for HCC patients with less than four liver tumors.

Table 4. Factors contributing to CR by DEB-TACE following cTACE (CR vs. non-CR).

\begin{tabular}{|c|c|c|c|c|c|c|c|c|}
\hline \multirow{3}{*}{$\begin{array}{c}\text { Parameters } \\
\text { Age }(<80 / 80 \leq)\end{array}$} & \multicolumn{4}{|c|}{ Uni-Variate Analysis } & \multicolumn{4}{|c|}{ Multi-Variate Analysis } \\
\hline & \multirow{2}{*}{$\begin{array}{c}\text { OR } \\
0.00772\end{array}$} & \multicolumn{2}{|c|}{$95 \% \mathrm{CI}$} & \multirow{2}{*}{$\frac{p \text {-Values }}{0.4717}$} & \multirow{2}{*}{$\begin{array}{c}\text { OR } \\
0.01196\end{array}$} & \multicolumn{2}{|c|}{$95 \% \mathrm{CI}$} & \multirow{2}{*}{$\begin{array}{c}p \text {-Values } \\
0.3896\end{array}$} \\
\hline & & -0.01404 & 0.02948 & & & -0.01671 & 0.04064 & \\
\hline Child-Pugh (A/B) & 0.02381 & -0.47115 & 0.51876 & 0.92187 & 0.10314 & -0.59448 & 0.80076 & 0.758 \\
\hline $\begin{array}{c}\text { Etiology } \\
\text { (HBVorHCV/NBNC) }\end{array}$ & 0.04546 & -0.373 & 0.46391 & 0.82479 & 0.05984 & -0.45035 & 0.57003 & 0.8068 \\
\hline Tumor number $(<4 / 4 \leq)$ & 0.50714 & 0.08651 & 0.92777 & *0.02009 & 0.69165 & 0.08865 & 1.29464 & ${ }^{*} 0.02716$ \\
\hline Tumor size $(<75 / 75 \leq)$ & 0.12143 & -0.34555 & 0.58841 & 0.59701 & 0.28535 & -0.31613 & 0.88683 & 0.32953 \\
\hline $\mathrm{MC}(\mathrm{SN}+\mathrm{SNE} / \mathrm{CMN})$ & -0.27174 & -0.84018 & 0.2967 & 0.33428 & 0.06001 & -0.72434 & 0.84435 & 0.8732 \\
\hline $\operatorname{AFP}(\mathrm{ng} / \mathrm{mL})(<9 / 9 \leq)$ & 0.1 & -0.4282 & 0.6282 & 0.69992 & -0.81159 & -3.33532 & 1.71213 & 0.50516 \\
\hline AFP-L3 $(\%)(<11 / 11 \leq)$ & 0.10989 & -0.29952 & 0.51930 & 0.58531 & 0.08614 & -0.37755 & 0.54983 & 0.6989 \\
\hline $\begin{array}{l}\mathrm{DCP}(\mathrm{mAU} / \mathrm{mL}) \\
(<3700 / 3700 \leq)\end{array}$ & 0.18681 & -0.21784 & 0.59147 & 0.35081 & 0.23685 & -0.32434 & 0.79804 & 0.3842 \\
\hline $\begin{array}{l}\text { cTACE (Cisplatin or } \\
\text { Miriplatin/Epirubicin) }\end{array}$ & -0.03846 & -0.45007 & 0.37314 & 0.84894 & 0.08178 & -0.41276 & 0.57632 & 0.7305 \\
\hline
\end{tabular}

Odds-ratios for continuous variables were calculated for one unit. $\left({ }^{*} p<0.05\right)$.

3.5. Comparison of Overall Survival Time between $C R$ and Non-CR $(P R+S D+P D)$ Patients in the cTACE Group

The comparison of the overall survival time between $\mathrm{CR}$ and non-CR (PR+SD+PD) patients in the cTACE group were analyzed. The median observation time was $14 \pm 13.1$ months after the first DEB-TACE. During the follow-up period, twenty-six patients $(96.3 \%)$ died and one patient $(3.7 \%)$ was still alive at the end of the observation. Among the patients with CR, thirteen patients (92.9\%) died and one patient $(7.1 \%)$ was still alive; among the cases with non-CR, thirteen patients $(100 \%)$ died (Figure 3$)$. Furthermore, there was a significant difference in the overall survival (OS) between CR and non-CR patients in the CTACE group $(* p=0.0403$, Figure 3$)$.

\subsection{Complications and Adverse Events}

One week after the additional cTACE, nine patients $(33.3 \%)$ experienced general fatigue, nine (33.3\%) experienced appetite loss, twelve (44.4\%) experienced fever, eight $(29.6 \%)$ experienced pain, two $(7.4 \%)$ had ascites, three $(11.1 \%)$ had anemia, five $(18.5 \%)$ had thrombocytopenia, four (14.8\%) had hyperbilirubinemia, and twenty-seven (100\%) had liver dysfunction, while only one (3.7\%) patient had severe thrombocytopenia (grade 3 ) (Table 5). One month after the additional cTACE, two patients $(7.4 \%)$ had ascites and no other adverse events were observed. 


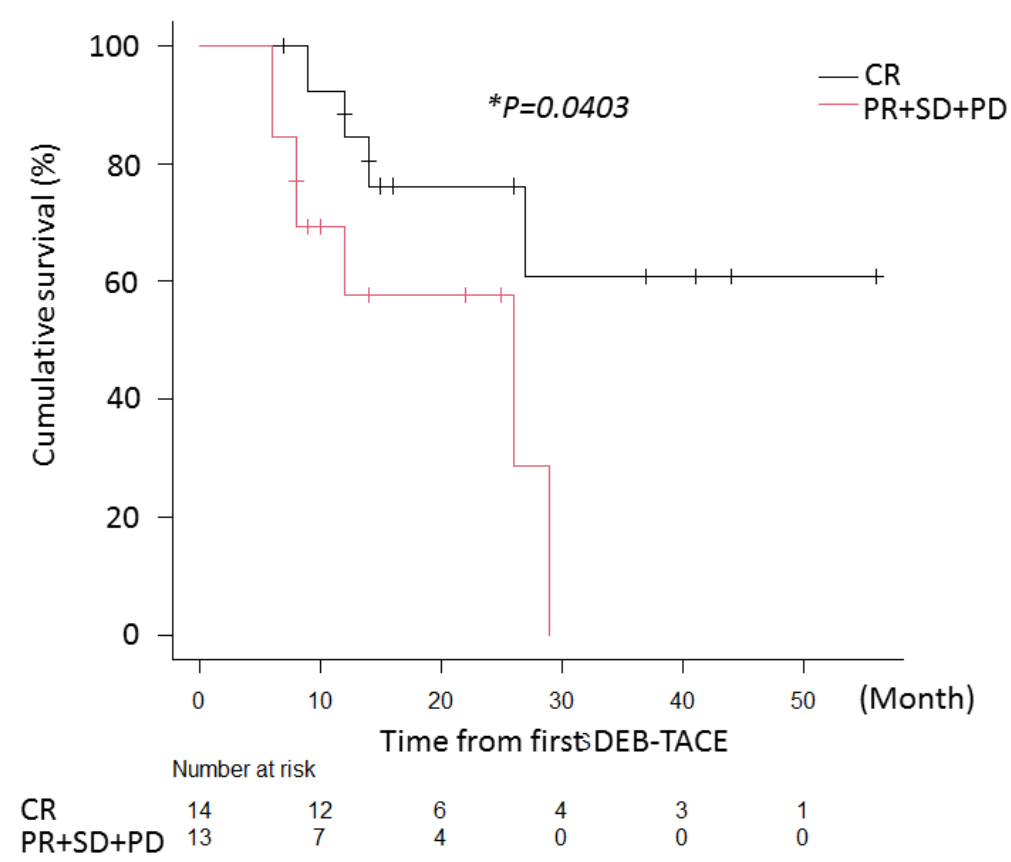

Figure 3. Cumulative rates of overall survival: the Kaplan-Meier method and log-rank test were used to assess the cumulative rates of overall survival. The black line indicates patients within the cTACE group and the red line indicates patients within the non-cTACE group $\left.{ }^{*} p<0.05\right)$.

Table 5. Safety profile following the additional cTACE $(n=27)$.

\begin{tabular}{ccc}
\hline CTCAE v5.0 & All Grades $\boldsymbol{n} \mathbf{( \% )}$ & Grade3 $\boldsymbol{n} \mathbf{( \% )}$ \\
\hline General fatigue & $9(33.3)$ & 0 \\
Appetite loss & $9(33.3)$ & 0 \\
Fever & $12(44.4)$ & 0 \\
Pain & $8(29.6)$ & 0 \\
Ascites & $2(7.4)$ & 0 \\
Anemia & $3(11.1)$ & 0 \\
Thrombocytopenia & $5(18.5)$ & $1(3.7)$ \\
Hyperbilirubinemia & $4(14.8)$ & 0 \\
Liver Dysfunction & $27(100)$ & 0
\end{tabular}

Data are presented as count $n$ (\%). Abbreviation: CTCAE v5.0, Common Terminology Criteria for Adverse Events version 5.0.

\section{Discussion}

Several clinical trials have demonstrated the efficacy and safety of DEB-TACE. A multicenter phase 2 randomized trial using DEB-TACE with doxorubicin-eluting beads (PRECISION V) showed a marked reduction in liver toxicity and drug-related adverse events as compared to that using cTACE with doxorubicin $[16,26,27]$. Our study also showed that DEB-TACE was efficient and safe (Table 2). Lammer et al. have also demonstrated that in patients with liver cancer, the tumor response is greater after DEB-TACE than cTACE $[26,28]$. Golfieri and colleagues conducted a randomized trial of DEB-TACE vs. CTACE in 177 patients with HCC. DEB-TACE and CTACE were found to be equally effective and safe, with the only advantage of DEB-TACE concerning the fact that it induced less postoperative abdominal pain [29]. The rationale behind DEB-TACE is that drug-loaded embolic microspheres help expose the target tumor to the antineoplastic drug for an extended period of time, while reducing the systemic circulation of the drug, resulting in lower toxicity for the patient [16]. Existing data therefore support our finding that DEB-TACE is useful for treating the intermediate stage of HCC. Nevertheless, there is no clinical evidence of DEB-TACE's possible advantage over cTACE. A randomized control study on this topic failed to show that DEB-TACE was superior to CTACE, with 
the two-year survival rates being $56.8 \%$ and $55.4 \%$, respectively [29]. Conversely, the CR rate of DEB-TACE was higher than that of CTACE, but the PR rate also remained high [30]. In addition, OS with DEB-TACE was not prolonged compared to OS with CTACE [31]. In our study, when we performed CTACE 2 months after DEB-TACE, the CR rate was clearly increased. Therefore, we hypothesized that the embolization sites of DEB-TACE and CTACE might be different, and this combination might be effective for the treatment of unresectable hepatocellular carcinoma. In fact, among several cases of liver tumor of the single or multiple nodular type, viable parts of the tumor are often detected after DEBTACE (Figure $2 b$ ). The remaining viable parts are due to the characteristics of DEB-TACE, regarding the fact that no drug-eluting beads reach the peribiliary vascular plexus [32]. This drainage area around the tumor is the first metastatic route of hepatocellular carcinoma and micrometastasis is detected at a high rate, and treatment similar to that of the cancerous part is desired [32]. In contrast, in cTACE, lipiodol injected from the hepatic artery fills the intratumoral sinus according to hemodyamics and accumulates in the drainage area [19]. We addressed that issue by performing an additional cTACE procedure by passing lipiodol through the vasculature to be accumulated in the tumor's drainage area (Figure 2f), which in turn might lead to an accumulation of lipiodol in the non-tumorous hepatic parenchyma around the liver tumor [19,33-36]. Our results demonstrated that DEB-TACE remarkably increased the complete response rate among the intermediate stage HCC patients (Table 3). Extrapolating from that finding, combined therapy with DEB-TACE followed by cTACE might also induce necrosis in the intermediate stage of HCC in a complementary manner.

Our data demonstrated that DEB-TACE followed by CTACE may be promising in the treatment of the intermediate stage of HCC, as it achieves a higher complete response rate and induces major tumor necrosis, while reducing the side effects of chemotherapy (Table 5). Additionally, the OS rate of CR patients was higher than that of non-CR patients in the cTACE group (Figure 3). In our study, we chose DEB-TACE as the first of two combined therapies because it is associated with less liver toxicity and fewer drug-related adverse events than CTACE, even though its therapeutic effect may be insufficient. In fact, Kalayci et al. showed that the area under the curve and peak concentration levels were the same between systemic chemotherapy and CTACE; thus, the value of CTACE is complicated by the side effects of chemotherapy [37]. With DEB-TACE, a lower amount of chemotherapeutic drugs pass through the patient's circulatory system, even when these drugs are locally injected in very high doses. Our study confirmed that the area under the curve and the peak concentration values produced by DEB-TACE were significantly lower than those produced by CTACE.

A therapeutic limitation of DEB-TACE concerns its possible insufficient embolization of the liver tumor. Drug-eluting beads are loaded into the peribiliary plexus, the main feeder of the bile duct wall. This could damage the area by decreasing arterial blood flow or by chemical insult to the vessel walls caused by highly concentrated antitumor drugs [32]. However, no drug-eluting beads accumulate in the tumor's drainage area, including non-tumorous hepatic parenchyma around the HCC. Therefore, cTACE may be critical for the treatment of the intermediate stage of HCC considering lipiodol in the non-tumorous liver adjacent to the tumor may correspond to the drainage area.

The present study has some limitations. The number of patients enrolled was limited and the follow-up duration was short. However, our findings concerning DEB-TACE followed by cTACE, which achieved a high CR rate and prolonged OS for CR patients as compared to non-CR patients in the cTACE group, are noteworthy. Therefore, our new combination therapy might be quite valuable for the treatment of the intermediate stage of HCC. Further research for the long-term outcomes with many participants might enable us to obtain the prolonged survival time of patients with intermediate stage HCC. 


\section{Conclusions}

DEB-TACE has recently been performed for intermediate stage HCC. However, the residual viable lesion located in the drainage area is often observed after DEB-TACE. CTACE involves accumulating lipiodol-containing anticancer drugs in the drainage area, which is the first site of HCC recurrence. Therefore, in our present study, DEB-TACE DEB-TACE followed by cTACE was able to reduce the side effects of chemotherapy while increasing the $\mathrm{CR}$ rate, which is only affected by the total number of tumors, and prolonge the OS of HCC patients who reached CR. This combination therapy appears to be promising for the treatment of the intermediate stage of HCC.

Author Contributions: A.M. (Asahiro Morishita) and T.N. conceived and designed the study. J.T., K.T. (Kei Takuma), M.N. (Mai Nakahara), K.O., T.T. (Tomoko Tadokoro), K.F. (Koji Fujita), T.S. (Tingting Shi), H.Y., T.M. (Takanori Matsui), T.T. (Tadayuki Takata), T.S. (Takayuki Sanomura), Y.N., T.H., T.T. (Tetsu Tomonari), A.M. (Akio Moriya), T.S. (Tomonori Senoo), and K.T. (Koichi Takaguchi). performed the data collection and analyzed the data. A.M. (Asahiro Morishita) and T.M. (Tsutomu Masaki) wrote the paper. All authors read and agreed to the published version of the manuscript.

Funding: No funding was received.

Institutional Review Board Statement: The present study conformed to the Clinical Research Guidelines and was approved by the ethical committee of Kagawa University, Faculty of Medicine (2019-271).

Informed Consent Statement: Not applicable.

Data Availability Statement: The data used in the present study are available from the corresponding author upon reasonable request.

Conflicts of Interest: All authors declare no conflict of interest.

\section{References}

1. Kim, J.Y.; Sinn, D.H.; Gwak, G.Y.; Choi, G.S.; Saleh, A.M.; Joh, J.W.; Cho, S.K.; Shin, S.W.; Carriere, K.C.; Ahn, J.H.; et al. Transarterial chemoembolization versus resection for intermediate-stage (BCLC B) hepatocellular carcinoma. Clin. Mol. Hepatol. 2016, 22, 250-258. [CrossRef]

2. Yang, B.; Li, C.L.; Guo, W.H.; Qin, T.Q.; Jiao, H.; Fei, Z.J.; Zhou, X.; Duan, L.J.; Liao, Z.Y. Intra-arterial ethanol embolization augments response to TACE for treatment of HCC with portal venous tumor thrombus. BMC Cancer 2018, 18, 101. [CrossRef]

3. Global Burden of Disease Cancer, C.; Fitzmaurice, C.; Akinyemiju, T.F.; Al Lami, F.H.; Alam, T.; Alizadeh-Navaei, R.; Allen, C.; Alsharif, U.; Alvis-Guzman, N.; Amini, E.; et al. Global, Regional, and National Cancer Incidence, Mortality, Years of Life Lost, Years Lived with Disability, and Disability-Adjusted Life-Years for 29 Cancer Groups, 1990 to 2016: A Systematic Analysis for the Global Burden of Disease Study. JAMA Oncol. 2018, 4, 1553-1568. [CrossRef]

4. Forner, A.; Reig, M.; Bruix, J. Hepatocellular carcinoma. Lancet 2018, 391, 1301-1314. [CrossRef]

5. Bray, F.; Ferlay, J.; Soerjomataram, I.; Siegel, R.L.; Torre, L.A.; Jemal, A. Global cancer statistics 2018: GLOBOCAN estimates of incidence and mortality worldwide for 36 cancers in 185 countries. CA Cancer J. Clin. 2018, 68, 394-424. [CrossRef]

6. Choo, S.P.; Tan, W.L.; Goh, B.K.P.; Tai, W.M.; Zhu, A.X. Comparison of hepatocellular carcinoma in Eastern versus Western populations. Cancer 2016, 122, 3430-3446. [CrossRef] [PubMed]

7. Ferenci, P.; Fried, M.; Labrecque, D.; Bruix, J.; Sherman, M.; Omata, M.; Heathcote, J.; Piratsivuth, T.; Kew, M.; Otegbayo, J.A.; et al. Hepatocellular carcinoma (HCC): A global perspective. J. Gastrointestin. Liver Dis. 2010, 19, 311-317. [CrossRef]

8. Malkowski, P.; Pacholczyk, M.; Lagiewska, B.; Adadynski, L.; Wasiak, D.; Kwiatkowski, A.; Chmura, A.; Czerwinski, J. Hepatocellular carcinoma-epidemiology and treatment. Przegl. Epidemiol. 2006, 60, 731-740. [PubMed]

9. Matsui, O.; Kadoya, M.; Yoshikawa, J.; Gabata, T.; Arai, K.; Demachi, H.; Miyayama, S.; Takashima, T.; Unoura, M.; Kogayashi, K. Small hepatocellular carcinoma: Treatment with subsegmental transcatheter arterial embolization. Radiology 1993, 188, 79-83. [CrossRef] [PubMed]

10. Kainuma, O.; Asano, T.; Aoyama, H.; Shinohara, Y. Recurrent hepatocellular carcinoma successfully treated with radiofrequency thermal ablation. J. Hepatobiliary Pancreat. Surg 1999, 6, 190-194. [CrossRef] [PubMed]

11. Katsanos, K.; Kitrou, P.; Spiliopoulos, S.; Maroulis, I.; Petsas, T.; Karnabatidis, D. Comparative effectiveness of different transarterial embolization therapies alone or in combination with local ablative or adjuvant systemic treatments for unresectable hepatocellular carcinoma: A network meta-analysis of randomized controlled trials. PLoS ONE 2017, 12, e0184597. [CrossRef] [PubMed]

12. Llovet, J.M.; Bru, C.; Bruix, J. Prognosis of hepatocellular carcinoma: The BCLC staging classification. Semin. Liver Dis. 1999, 19, 329-338. [CrossRef] 
13. Raoul, J.L.; Forner, A.; Bolondi, L.; Cheung, T.T.; Kloeckner, R.; de Baere, T. Updated use of TACE for hepatocellular carcinoma treatment: How and when to use it based on clinical evidence. Cancer Treat. Rev. 2019, 72, 28-36. [CrossRef] [PubMed]

14. Kobayashi, S.; Tajiri, K.; Murayama, A.; Entani, T.; Futsukaichi, Y.; Nagata, K.; Takahashi, K.; Yasuda, I. Drug-eluting BeadTranscatheter Arterial Chemoembolization for Advanced Hepatocellular Carcinoma Refractory to Conventional Lipiodol-based Transcatheter Arterial Chemoembolization. J. Hepatocell. Carcinoma 2020, 7, 181-189. [CrossRef]

15. Chen, P.; Yuan, P.; Chen, B.; Sun, J.; Shen, H.; Qian, Y. Evaluation of drug-eluting beads versus conventional transcatheter arterial chemoembolization in patients with unresectable hepatocellular carcinoma: A systematic review and meta-analysis. Clin. Res. Hepatol. Gastroenterol. 2017, 41, 75-85. [CrossRef]

16. Varela, M.; Real, M.I.; Burrel, M.; Forner, A.; Sala, M.; Brunet, M.; Ayuso, C.; Castells, L.; Montana, X.; Llovet, J.M.; et al. Chemoembolization of hepatocellular carcinoma with drug eluting beads: Efficacy and doxorubicin pharmacokinetics. J. Hepatol. 2007, 46, 474-481. [CrossRef] [PubMed]

17. Nakano, M.M.; Yamamoto, A.; Nishida, N.; Hamuro, M.; Hamamoto, S.; Jogo, A.; Sohgawa, E.; Kageyama, K.; Minami, T.; Miki, Y. Risk factors for local recurrence of hepatocellular carcinoma after transcatheter arterial chemoembolization with drug-eluting beads (DEB-TACE). Jpn. J. Radiol. 2019, 37, 543-548. [CrossRef]

18. Peng, Z.; Cao, G.; Hou, Q.; Li, L.; Ying, S.; Sun, J.; Zhou, G.; Zhou, J.; Zhang, X.; Ji, W.; et al. The comprehensive analysis of efficacy and safety of CalliSpheres(R) drug-eluting beads transarterial chemoembolization in 367 liver cancer patients: A multiplecenter, cohort study. Oncol. Res. 2019, 28, 249-271. [CrossRef]

19. Terayama, N.; Matsui, O.; Gabata, T.; Kobayashi, S.; Sanada, J.; Ueda, K.; Kadoya, M.; Kawamori, Y. Accumulation of iodized oil within the nonneoplastic liver adjacent to hepatocellular carcinoma via the drainage routes of the tumor after transcatheter arterial embolization. Cardiovasc. Interv. Radiol. 2001, 24, 383-387. [CrossRef]

20. Taketa, K.; Endo, Y.; Sekiya, C.; Tanikawa, K.; Koji, T.; Taga, H.; Satomura, S.; Matsuura, S.; Kawai, T.; Hirai, H. A collaborative study for the evaluation of lectin-reactive alpha-fetoproteins in early detection of hepatocellular carcinoma. Cancer Res. 1993, $53,5419-5423$.

21. Murakami, N.; Tamano, M.; Yoneda, M.; Sugaya, H.; Hiraishi, H. Des-gamma-carboxy prothrombin (DCP) ratio is a useful prognostic tumor marker for single nodule hepatocellular carcinoma (HCC). Hepatogastroenterology 2008, 55, 197-201. [PubMed]

22. Shiozawa, K.; Watanabe, M.; Ikehara, T.; Ogino, Y.; Umakoshi, T.; Matsukiyo, Y.; Kogame, M.; Matsui, T.; Kikuchi, Y.; Igarashi, Y.; et al. Delayed intratumoral hemorrhage after drug-eluting bead transarterial chemoembolization for hepatocellular carcinoma. Case Rep. Oncol. 2014, 7, 739-745. [CrossRef] [PubMed]

23. Wiggermann, P.; Sieron, D.; Brosche, C.; Brauer, T.; Scheer, F.; Platzek, I.; Wawrzynek, W.; Stroszczynski, C. Transarterial Chemoembolization of Child-A hepatocellular carcinoma: Drug-eluting bead TACE (DEB TACE) vs. TACE with cisplatin/lipiodol (cTACE). Med. Sci. Monit. 2011, 17, CR189-CR195. [CrossRef]

24. Kudo, M.; Ueshima, K.; Kubo, S.; Sakamoto, M.; Tanaka, M.; Ikai, I.; Furuse, J.; Murakami, T.; Kadoya, M.; Kokudo, N.; et al. Response Evaluation Criteria in Cancer of the Liver (RECICL) (2015 Revised version). Hepatol. Res. 2016, 46, 3-9. [CrossRef]

25. Trotti, A.; Colevas, A.D.; Setser, A.; Basch, E. Patient-reported outcomes and the evolution of adverse event reporting in oncology. J. Clin. Oncol. 2007, 25, 5121-5127. [CrossRef]

26. Lammer, J.; Malagari, K.; Vogl, T.; Pilleul, F.; Denys, A.; Watkinson, A.; Pitton, M.; Sergent, G.; Pfammatter, T.; Terraz, S.; et al. Prospective randomized study of doxorubicin-eluting-bead embolization in the treatment of hepatocellular carcinoma: Results of the PRECISION V study. Cardiovasc. Interv. Radiol. 2010, 33, 41-52. [CrossRef]

27. Vogl, T.J.; Lammer, J.; Lencioni, R.; Malagari, K.; Watkinson, A.; Pilleul, F.; Denys, A.; Lee, C. Liver, gastrointestinal, and cardiac toxicity in intermediate hepatocellular carcinoma treated with PRECISION TACE with drug-eluting beads: Results from the PRECISION V randomized trial. AJR Am. J. Roentgenol. 2011, 197, W562-W570. [CrossRef]

28. Ikeda, K. Recent advances in medical management of hepatocellular carcinoma. Hepatol. Res. 2019, 49, 14-32. [CrossRef] [PubMed]

29. Golfieri, R.; Giampalma, E.; Renzulli, M.; Cioni, R.; Bargellini, I.; Bartolozzi, C.; Breatta, A.D.; Gandini, G.; Nani, R.; Gasparini, D.; et al. Randomised controlled trial of doxorubicin-eluting beads vs conventional chemoembolisation for hepatocellular carcinoma. Br. J. Cancer 2014, 111, 255-264. [CrossRef]

30. Li, H.; Wu, F.; Duan, M.; Zhang, G. Drug-eluting bead transarterial chemoembolization (TACE) vs conventional TACE in treating hepatocellular carcinoma patients with multiple conventional TACE treatments history: A comparison of efficacy and safety. Medicine 2019, 98, e15314. [CrossRef] [PubMed]

31. Kloeckner, R.; Weinmann, A.; Prinz, F.; Pinto dos Santos, D.; Ruckes, C.; Dueber, C.; Pitton, M.B. Conventional transarterial chemoembolization versus drug-eluting bead transarterial chemoembolization for the treatment of hepatocellular carcinoma. BMC Cancer 2015, 15, 465. [CrossRef] [PubMed]

32. Guiu, B.; Deschamps, F.; Aho, S.; Munck, F.; Dromain, C.; Boige, V.; Malka, D.; Leboulleux, S.; Ducreux, M.; Schlumberger, M.; et al. Liver/biliary injuries following chemoembolisation of endocrine tumours and hepatocellular carcinoma: Lipiodol vs. drug-eluting beads. J. Hepatol. 2012, 56, 609-617. [CrossRef] [PubMed]

33. Ueda, K.; Matsui, O.; Kawamori, Y.; Nakanuma, Y.; Kadoya, M.; Yoshikawa, J.; Gabata, T.; Nonomura, A.; Takashima, T. Hypervascular hepatocellular carcinoma: Evaluation of hemodynamics with dynamic CT during hepatic arteriography. Radiology 1998, 206, 161-166. [CrossRef] [PubMed] 
34. Matsuo, N.; Uchida, H.; Nishimine, K.; Soda, S.; Oshima, M.; Nakano, H.; Nagano, N.; Nishimura, Y.; Yoshioka, T.; Guo, Q.; et al. Segmental transcatheter hepatic artery chemoembolization with iodized oil for hepatocellular carcinoma: Antitumor effect and influence on normal tissue. J. Vasc. Interv. Radiol. 1993, 4, 543-549. [CrossRef]

35. Nakamura, H.; Hashimoto, T.; Oi, H.; Sawada, S. Iodized oil in the portal vein after arterial embolization. Radiology 1988, 167, 415-417. [CrossRef]

36. Nakamura, H.; Hashimoto, T.; Oi, H.; Sawada, S. Transcatheter oily chemoembolization of hepatocellular carcinoma. Radiology 1989, 170 Pt 1, 783-786. [CrossRef]

37. Kalayci, C.; Johnson, P.J.; Raby, N.; Metivier, E.M.; Williams, R. Intraarterial adriamycin and lipiodol for inoperable hepatocellular carcinoma: A comparison with intravenous adriamycin. J. Hepatol. 1990, 11, 349-353. [CrossRef] 